\title{
Decreased expression of the long non-coding RNA MLLT4 antisense RNA 1 is a potential biomarker and an indicator of a poor prognosis for gastric cancer
}

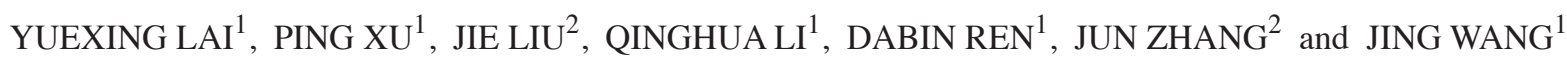 \\ ${ }^{1}$ Department of Gastroenterology, Shanghai Songjiang Hospital Affiliated to Nanjing Medical University, Shanghai 201600; \\ ${ }^{2}$ Department of Digestive Diseases, Huashan Hospital, Fudan University, Shanghai 200040, P.R. China
}

Received June 5, 2015; Accepted October 14, 2016

DOI: $10.3892 / 01.2017 .6478$

\begin{abstract}
In recent years, the identification of long non-coding RNAs (lncRNAs) led to the analysis of their characteristics in cancer biology. However, the expression of lncRNAs in cancer and their clinical significance remain unclear. In the present study, an investigation of lncRNAs that may be involved in the regulation of metastasis using microarray and polymerase chain reaction analyses resulted in the identification of MLLT4 antisense RNA 1 (MLLT4-AS1) as a significantly downregulated lncRNA in gastric cancer tissue compared with normal adjacent tissue $(\mathrm{P}=0.006)$. Furthermore, the downregulation of MLL4-AS1 was significantly associated with advanced Tumor-Node-Metastasis stage $(\mathrm{P}=0.007)$ and lymph node metastasis $(\mathrm{P}=0.008)$. Cox regression analysis showed that MLLT4-AS1 expression was an independent predictor for overall survival (hazard ratio, 13.136; 95\% confidence interval, 5.065-34.068; $\mathrm{P}<0.001)$. These data suggest that the decreased expression of MLLT4-AS1 is a potential biomarker and a predictor of a poor prognosis for gastric cancer.
\end{abstract}

\section{Introduction}

Gastric cancer is the fourth most commonly occurring malignancy and the second leading cause of cancer-associated mortality worldwide (1). Almost two-thirds of gastric cancer cases occur in developing countries, with an incidence of $\sim 42 \%$ in China alone (2). Despite improvements in therapy in the past decades, this type of cancer remains highly lethal due to its aggressive metastatic behavior and the fact that it is often diagnosed at an advanced stage (3). An improved understanding of the disease-causing mechanism and the

Correspondence to: Dr Ping Xu, Department of Gastroenterology, Shanghai Songjiang Hospital Affiliated to Nanjing Medical University, 746 Middle Zhongshan Road, Songjiang, Shanghai 201600, P.R. China

E-mail: yfyxp@aliyun.com

Key words: long non-coding RNA, MLLT4-AS1, gastric cancer identification of specific biomarkers for gastric cancer progression are urgently required for the prediction and improvement of clinical outcomes.

Human genome studies have identified a large number of non-coding RNAs (ncRNAs) that are differentially-expressed in varying organs and tissue types (4-7). Such developments have been equaled through discoveries made by analyzing the role of ncRNAs in human diseases, particularly cancer, which has corroborated the importance of their cellular functions $(8,9)$. Preliminary results have indicated that ncRNAs, particularly long ncRNAs (lncRNA), exhibit key roles in tumorigenesis (8), and that lncRNA-mediated biology is focal to the progression of cancer $(8,10-13)$. Those lncRNAs associated with cancer are often aberrantly expressed and affect cancer progression through different mechanisms $(14,15)$. Therefore, a better understanding of the expression and function of IncRNAs may lead to the identification of novel biomarkers and therapeutic targets for the treatment of cancer.

The present primary investigation of lncRNAs that may be involved in gastric cancer progression led to the identification of several noteworthy candidates. One of these was MLLT4 antisense RNA 1 (MLLT4-AS1), which is also known as chromosome 6 open reading frame 124 (C6orf124), dJ431P23.3 or HGC6.4. This gene is located in chromosome 6:167,823,876-167,826,709, and 3 transcripts (splice variants) have been identified, namely MLLT4-AS1-001 (2,238 bp), MLLT4-AS1-002 (311 bp) and MLLT4-AS1-003 (182 bp) (www.ensembl.org). It is unknown whether this gene is associated with cancer. In the present study, the expression level of MLLT4-AS1 was examined in gastric cancer tissues and the potential correlation between its expression level and the clinicopathological features of gastric cancer patients was evaluated. These findings indicated that decreased expression of MLLT4-AS1 is associated with a poor prognosis in gastric cancer.

\section{Materials and methods}

Sample preparation. A total of 103 human primary gastric cancer samples and paired adjacent non-cancerous tissue samples were collected after obtaining informed consent from patients who underwent D2 radical resection between 
January 2007 and December 2008 in Shanghai Songjiang Hospital Affiliated to Nanjing Medical University (Shanghai, China). Of these, 5 tissue samples were randomly selected for human IncRNA microarray analysis and the remaining 98 were used for quantitative polymerase chain reaction (qPCR) analysis. The study was approved by the Ethics Committee of the Shanghai Songjiang Hospital Affiliated to Nanjing Medical University. All subjects provided informed written consent at the time of surgery for donation of their tissue for this study. Specimens were obtained immediately after surgical resection and stored at $-80^{\circ} \mathrm{C}$ for further analysis. Lymph nodes (LNs) with or without metastasis were also harvested during gastrectomy. The 98 samples analyzed by qPCR were obtained from 51 men and 47 women, with a median age of 57 years (range, 31-83 years). Tumor stage was defined according to the American Joint Committee on Cancer/International Union against Cancer Tumor-Node-Metastasis (TNM) classification system (seventh edition) (16). Clinical data, including date of birth, sex, date of surgery, serum carcinoembryonic antigen (CEA) level, Helicobacter pylori status, tumor size, tumor location and other content of histopathological reports, were extracted from the computerized clinical database.

RNA preparation. RNA preparation. Briefly, gastric cancer and paired adjacent non-cancerous tissues were homogenized in TRIzol reagent $(1 \mathrm{ml}$ per 50-100 mg tissue; Invitrogen; Thermo Fisher Scientific, Inc., Waltham, MA, USA). After sample homogenization, total RNA was extracted following the manufacturer's instructions. The concentration and quality of total RNA from each sample were measured using a NanoDrop ND-1000 (Thermo Fisher Scientific, Inc.), and RNA integrity was assessed by $1.5 \%$ agarose-formaldehyde gel electrophoresis.

lncRNA and mRNA microarray. The Human lncRNA 4*180K array was manufactured by Agilent Technologies, Inc. (Santa Clara, CA, USA). Each array represented all long transcripts, including protein coding mRNAs and IncRNAs in the human genome. More than 41,053 lncRNAs were collected. Each transcript was represented by $1-5$ probes to improve statistical confidence.

Microarray analysis. For microarray analysis, RNA purity and integrity was analyzed by Agilent Bioanalyzer 2100 (Agilent Technologies, Inc.). Qualified total RNA was further purified by RNeasy mini kit (Qiagen, Hilden, Germany) and RNase-free DNase set (Qiagen). Total RNA was then amplified and labeled by a Low Input Quick Amp Labeling kit, One-Color (Agilent), following the manufacturer's instructions. Labeled cRNA were purified by RNeasy mini kit (Qiagen). Each Slide was hybridized with $600 \mathrm{ng}$ Cy3-labeled cRNA using a Gene Expression Hybridization kit (Agilent Technologies, Inc.) in a Hybridization Oven (Agilent Technologies, Inc.), according to the manufacturer's instructions. After $17 \mathrm{~h}$ of hybridization, the slides were washed in staining dishes (Thermo Fisher Scientific, Inc.) with Gene Expression Wash Buffer kit (Agilent Technologies, Inc.), following the manufacturer's instructions. Slides were scanned by Agilent Microarray Scanner (Agilent) with default settings as follows: Dye channel, green; scan resolution, $3 \mu \mathrm{m}$; 20 bit. Data were extracted with Feature Extraction software 10.7 (Agilent Technologies, Inc.). Raw data were normalized by Quantile algorithm, Gene Spring Software 11.0 (Agilent).

Reverse transcription ( $R T)-q P C R$. The mRNA from gastric cancer samples and paired adjacent non-cancerous tissues was analyzed by reverse transcription using M-MLV Reverse Transcriptase (Takara Biotechnology, Co., Ltd., Dalian, China). The cDNA template was amplified by RT-qPCR using the SYBR ${ }^{\circledR}$ Premix Dimmer Eraser kit (Takara Biotechnology, Co., Ltd.). Primer sequences used for MLLT4-AS1 amplifications were as follows: Forward, 5'-TGCTGTGCGGTGTTCCTCTC-3' and reverse, 5'-CGAAGAATTGGCAGATAACGATGT-3'. Glyceraldehyde 3-phosphate dehydrogenase (GAPDH) was used as an internal control (forward, 5'-ACCCACTCCTCCACCTTT GAC-3' and reverse, 5'-TGTTGCTGTAGCCAAATTCGT T-3'), and MLLT4-AS1 values were normalized to GAPDH. RT-qPCR was performed with the ABI7500 system (Applied Biosystems; Thermo Fisher Scientific, Inc.). The cycling conditions were as follows: $95^{\circ} \mathrm{C}$ for $10 \mathrm{~min}$, followed by 40 cycles of $95^{\circ} \mathrm{C}$ for $15 \mathrm{sec}$ and $60^{\circ} \mathrm{C}$ for $45 \mathrm{sec}$. All experiments were repeated 3 times. The relative expression fold-change of the mRNA was calculated using the $2^{-\Delta \Delta C q}$ method (17).

Statistical analysis. Comparisons of continuous data between the two groups were performed with the independent $t$-test or paired t-test, whereas categorical data were analyzed using the $\chi^{2}$ test. Overall survival was analyzed by the Kaplan-Meier method, and the differences between groups were estimated by the log-rank test. Independent prognostic indicators were assessed by multivariate analysis using Cox's proportional hazards regression model. All statistical analyses were performed using SPSS for Windows v.16.0 (SPSS, Inc., Chicago, IL, USA) and GraphPad Prism 5.0 (GraphPad Software, La Jolla, CA, USA). P $<0.05$ was considered to indicate a statistically significant difference.

\section{Results}

IncRNAs are aberrantly expressed in gastric cancer compared with adjacent non-cancerous tissues. To investigate the potential biological functions of lncRNAs in gastric cancer, the lncRNA expression profiles in human gastric cancer were examined using microarray analysis. The lncRNA expression profiling data revealed 41,053 lncRNAs expressed in gastric cancer (Fig. 1); of these, 1,102 lncRNAs showed different expression profiles (fold-change, $\geq 2.0$ or $\leq 0.5 ; \mathrm{P}<0.01$ ) between the gastric cancer and adjacent non-cancerous tissues. Among these, 448 lncRNAs were upregulated and 654 were downregulated in the gastric cancer tissues compared with the adjacent non-cancerous tissues. MLLT4-AS1 was significantly downregulated (fold-change, 0.48).

MLLT4-AS1 is downregulated in human gastric carcinoma tissues. The expression of MLLT4-AS1, which was identified as a significantly downregulated lncRNA in gastric cancer, was further examined in 98 pairs of human gastric cancer and adjacent non-cancerous tissues using qPCR. Downregulation of MLLT4-AS1 was detected in 77/98 (78.6\%) gastric 
A

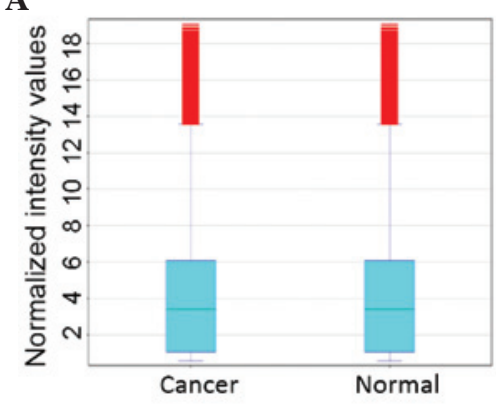

B

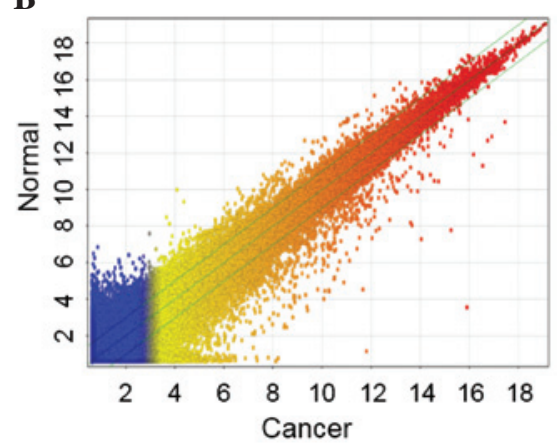

Figure 1. IncRNA and mRNA profile comparison between gastric cancer samples and adjacent non-cancerous tissues. (A) The box plot is a convenient way to visualize the distribution of a dataset in the lncRNA profiles. After normalization, the distributions of $\log 2$-ratios among the tested samples are nearly the same. (B) The scatter-plot is used for assessing lncRNA expression variation between gastric cancer samples and adjacent non-cancerous tissues. The $\mathrm{X}$ and $\mathrm{Y}$ axes in the scatter-plot represent averaged normalized values in each group (log2 scaled). The green lines represent fold-changes (the default fold-change value was 3.0). The lncRNAs above the top green line and below the bottom green line indicate $>3$-fold change of lncRNAs between pairs. lncRNA, long non-coding RNA.
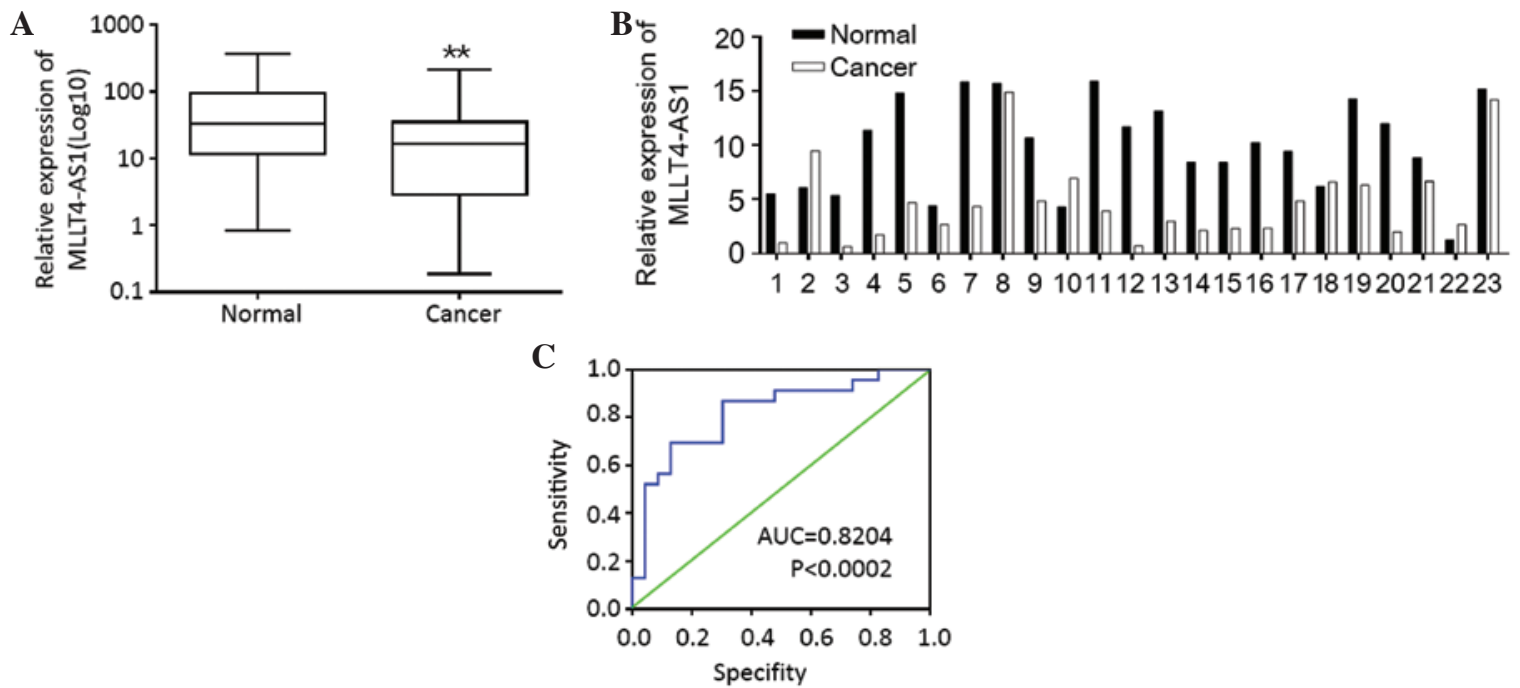

Figure 2. MLLT4-AS1 expression in gastric cancer tissues and its clinical significance. (A) The relative expression of MLLT4-AS1 was quantified by reverse transcription-qunatitative polymerase chain reaction in tumorous and adjacent non-tumorous tissues. (B) Relative expression of MLLT4-AS1 in LNs with or without metastasis. (C) Receiver operating characteristic analysis of MLLT4-AS1 expression for the prediction of lymph node metastasis. ${ }^{* *} \mathrm{P}<0.01$ vs. control. MLLT4-AS1, MLLT4 antisense RNA 1.

cancer samples compared with their non-tumorous counterparts $(\mathrm{P}=0.006$; Fig. $2 \mathrm{~A})$, indicating that MLLT4-AS1 was frequently downregulated in gastric cancer.

Next, the association between MLLT4-AS1 expression and various clinicopathological parameters was evaluated. Low MLLT4-AS1 expression was positively correlated with advanced TNM stage $(\mathrm{P}=0.007)$ and $\mathrm{LN}$ metastasis $(\mathrm{P}=0.008)$. No significant correlation was observed between MLLT4-AS1 expression and sex, age, location of tumor, size of tumor, liver metastasis, Lauren's classification or serum CEA levels (Table I).

Downregulation of MLLT4-AS1 is associated with LN metastasis. LN metastasis is one of the most important prognostic factors in patients with gastric cancer. To further investigate the role of MLLT4-AS1 in LN metastasis, MLLT4-AS1 expression was compared between 23 paired LN specimens using RT-qPCR. Each paired LN specimen consisted of one

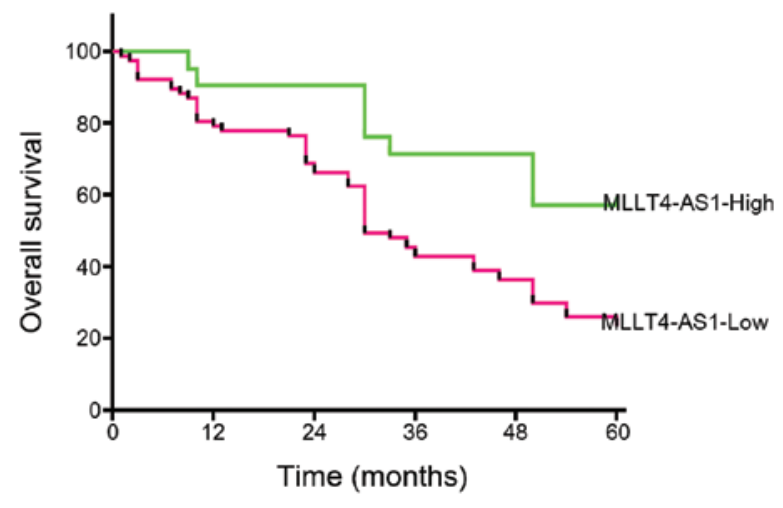

Figure 3. Overall survival curves of patients with gastric cancer according to MLLT4-AS1 expression levels. MLLT4-AS1, MLLT4 antisense RNA 1.

LN with metastasis and one without metastasis, derived from the same patient. Overall, 19/23 pairs of LNs (82.6\%) showed 
Table I. Association between MLLT4-AS1 expression and clinicopathological features.

\begin{tabular}{|c|c|c|c|c|c|}
\hline \multirow[b]{2}{*}{ Clinicopathological variable } & \multirow[b]{2}{*}{$\mathrm{n}$} & \multicolumn{2}{|c|}{ MLLT4-AS1 expression } & \multirow[b]{2}{*}{$\chi^{2}$} & \multirow[b]{2}{*}{ P-value } \\
\hline & & Low & High & & \\
\hline All cases & 98 & 77 & 21 & & \\
\hline Age, years & & & & 0.766 & 0.381 \\
\hline$\leqq 50$ & 36 & 30 & 6 & & \\
\hline$>50$ & 62 & 47 & 15 & & \\
\hline Sex & & & & 0.001 & 0.972 \\
\hline Male & 51 & 40 & 11 & & \\
\hline Female & 47 & 37 & 10 & & \\
\hline $\mathrm{HP}$ & & & & 1.787 & 0.181 \\
\hline Positive & 50 & 42 & 8 & & \\
\hline Negative & 48 & 35 & 13 & & \\
\hline Size of tumor, $\mathrm{cm}$ & & & & 1.265 & 0.261 \\
\hline$<5$ (small) & 32 & 23 & 9 & & \\
\hline$\geqq 5$ (large) & 66 & 54 & 12 & & \\
\hline Location of tumor & & & & 1.874 & 0.392 \\
\hline Cardia & 22 & 15 & 7 & & \\
\hline Body & 25 & 20 & 5 & & \\
\hline Antrum & 51 & 42 & 9 & & \\
\hline Depth of tumor invasion & & & & 0.466 & 0.495 \\
\hline $\mathrm{T} 1-\mathrm{T} 2$ & 39 & 32 & 7 & & \\
\hline $\mathrm{T} 3-\mathrm{T} 4$ & 59 & 45 & 14 & & \\
\hline Lymph node metastasis & & & & 7.052 & 0.008 \\
\hline Present & 75 & 64 & 11 & & \\
\hline Absent & 23 & 13 & 10 & & \\
\hline Liver metastasis & & & & 0.429 & 0.513 \\
\hline Absent & 69 & 53 & 16 & & \\
\hline Present & 29 & 24 & 5 & & \\
\hline Invasion of contiguous organs & & & & 3.655 & 0.056 \\
\hline Yes & 26 & 17 & 9 & & \\
\hline No & 72 & 60 & 12 & & \\
\hline Vessel invasion & & & & 0.839 & 0.360 \\
\hline Negative & 52 & 39 & 13 & & \\
\hline Positive & 46 & 38 & 8 & & \\
\hline Stage & & & & 7.289 & 0.007 \\
\hline I, II & 32 & 20 & 12 & & \\
\hline III, IV & 66 & 57 & 9 & & \\
\hline Lauren's classification & & & & 0.705 & 0.401 \\
\hline Diffuse & 30 & 22 & 8 & & \\
\hline Intestinal & 68 & 55 & 13 & & \\
\hline Grade of differentiation & & & & 1.767 & 0.184 \\
\hline Well and moderate & 39 & 28 & 11 & & \\
\hline Poor and undifferentiated & 59 & 49 & 10 & & \\
\hline Pre-operative chemotherapy & & & & 1.445 & 0.229 \\
\hline Yes & 44 & 37 & 7 & & \\
\hline No & 54 & 40 & 14 & & \\
\hline Serum CEA value, $\mu \mathrm{g} / 1$ & & & & 0.105 & 0.746 \\
\hline$<5$ & 59 & 47 & 12 & & \\
\hline$\geq 5$ & 39 & 30 & 9 & & \\
\hline
\end{tabular}


Table II. Univariate and multivariate analyses of factors associated with overall survival.

\begin{tabular}{lcccr}
\hline & & & Multivariate \\
\cline { 3 - 5 } Clinicopathological variable & $\begin{array}{c}\text { Univariate } \\
\text { P-value }\end{array}$ & Hazard ratio & $95 \%$ CI & P-value \\
\hline Age: $\leq 50$ vs. $>$ 50 years & 0.301 & 0.914 & $0.349-2.393$ & 0.855 \\
Sex: Male vs. female & 0.342 & 1.303 & $0.708-2.393$ & 0.396 \\
HP: Positive vs. negative & 0.280 & 0.824 & $0.459-1.480$ & 0.518 \\
Size: $<5$ vs. 5 cm & 0.262 & 0.962 & $0.542-1.707$ & 0.893 \\
Location: Cardia vs. body vs. antrum & 0.324 & 1.164 & $0.935-1.449$ & 0.173 \\
Invasion depth: T1-T2 vs. T3-T4 & 0.550 & 0.824 & $0.457-1.488$ & 0.522 \\
LNM: N0 vs. N1 vs. N2 vs. N3a vs. N3b & $<0.001$ & 4.330 & $1.572-11.930$ & 0.005 \\
Liver metastasis: Yes vs. no & 0.254 & 1.192 & $0.633-2.245$ & 0.586 \\
MLLT4-AS1: High vs. low & $<0.001$ & 13.136 & $5.065-34.068$ & $<0.001$ \\
Invasion of contiguous organs: Yes vs. no & 0.869 & 0.684 & $0.356-1.314$ & 0.254 \\
Microvessel invasion: Yes vs. no & 0.823 & 1.156 & $0.676-1.977$ & 0.596 \\
Stage: I, II vs. III, IV & $<0.001$ & 6.489 & $2.932-14.360$ & $<0.001$ \\
Lauren's classification: Diffuse vs. intestinal & 0.618 & 0.724 & $0.371-1.416$ & 0.724 \\
Grade of differentiation: Well and moderate vs. poor & 0.650 & 0.960 & $0.534-1.725$ & 0.892 \\
Preoperative chemotherapy: Yes vs. no & 0.030 & 1.100 & $0.613-1.974$ & 0.750 \\
CEA: 5 vs. $>5$ 5 $\mu$ glml & 0.797 & 0.660 & $0.376-1.158$ & 0.147 \\
\hline
\end{tabular}

MLLT4-AS1, MLLT4 antisense RNA 1; LNM, lymph node metastasis; CEA, carcinoembryonic antigen; CI, confidence interval; HP, Helicobacter pylori.

lower MLLT4-AS1 expression in the metastatic LNs than in their matched non-metastatic counterparts ( $\mathrm{P}=0.017$; Fig. 2B).

In addition, the study investigated whether MLLT4-AS1 expression status in the primary tumor could predict the presence of LN metastasis. Calculation of predictive values by receiver operating curve analysis showed that the area under the curve was 0.8204 (Fig. 2C).

MLLT4-AS1 expression and clinical outcomes. The 1-, 3- and 5-year cumulative survival rates for patients with high MLLT4-AS1 expression were 90, 71 and 57\% respectively, whereas the corresponding values for patients with low MLLT4-AS1 expression were 78, 43 and 23\%, respectively. These results indicated that gastric cancer patients with low MLLT4-AS1 expression had a poorer prognosis than those with high MLLT4-AS1 expression $(\mathrm{P}<0.05$; Fig. 3). Potential prognostic factors of 98 cases gastric cancer patients were analyzed by the Cox's proportional hazards regression model to investigate the association between patient survival and several clinicopathological parameters (Table II). The results indicated that MLLT4-AS1 expression was an independent prognostic factor for patients with gastric cancer [Hazard ratio (HR), 13.136; 95\% CI, 5.065-34.068; $\mathrm{P}<0.001]$, in addition to the TNM stage (HR, 6.489; 95\% CI, 2.932-14.360; $\mathrm{P}<0.001)$ and LN metastasis (HR, 4.330; 95\% CI, 1.572-11.930; $\mathrm{P}=0.005)$ (Table II).

\section{Discussion}

The present study showed for the first time that the lncRNA MLLT4-AS1 is downregulated in gastric cancer tissues. The downregulation of MLL4-AS1 expression was significantly associated with histological grade, LN metastasis, distant metastasis and a shorter disease-free interval. These data suggested that MLLT4-AS1 functions as a tumor suppressor gene and that downregulation of MLLT4-AS1 is a potential predictor of a poor disease prognosis.

Two issues remain to be addressed. Firstly, the mechanism by which MLLT4-AS1 is silenced in gastric cancer. In cancer cells, tumor suppressive genes are usually silenced by genetic (18) and epigenetic (19) alterations. Two main pathways are involved in the process of genetic alteration. One pathway is the hypermutability pathway, in which repair gene inactivation results in an increased mutation rate, affecting a number of different genes (20) and leading to deregulated cancer cell proliferation. In the second pathway, the chromosomal instability pathway, gross chromosomal alterations result in aneuploidy of cancer cells and lead to tumor suppressor gene inactivation and oncogene activation (21). Studies have reported that chromosome 6 is a target of chromosome instability that is associated with gastric cancer development. Deletions of the long arm of chromosome 6 have been observed in 26-45\% of primary gastric carcinomas (22-26). Two regions on chromosome 6 undergo heterozygous loss in primary gastric carcinomas; the region between $6 \mathrm{q} 16.3$ and $6 \mathrm{q} 23$ is lost in $50 \%$ of informative cases, whereas the region between $6 \mathrm{q} 26$ and $6 q 27$ is lost in $37 \%$ of informative cases (27). MLLT4-AS1 is located in 6q27 (www.ensembl.org), which indicates that the silencing of MLLT4-AS1 in gastric cancer may result from the heterozygous loss of regions on chromosome 6 . However, the possibility that epigenetic alterations may also play a role cannot be excluded.

The second issue to be addresses is the mechanism linking MLLT4-AS1 loss to enhanced gastric cancer metastasis. 
To date, the majority of well-characterized lncRNAs have exhibited a functional role in gene expression regulation, and normally in transcriptional rather than post-transcriptional regulation. This may occur through the targeting of genomically local (cis-regulation) or genomically distant (trans-regulation) genes (28). Typically, antisense lncRNAs regulate gene transcription by suppressing the expression of their sense counterparts (29). The counterpart of MLLT4-AS1 is MLLT4, which encodes afadin/AF6, an actin-binding protein that regulates cell-cell adhesions. Previous studies have revealed an association between afadin/AF6 and cancer (30-32). For instance, loss of afadin/AF6 expression, which is associated with adverse prognosis and increased risk of metastatic relapse in breast cancer, induces cell migration, invasiveness, and tumor growth (33). Nevertheless, in future studies, it would be of interest to investigate whether the role of MLLT4-AS1 in gastric cancer metastasis involves the regulation of the expression of its sense counterpart.

In summary, the present study showed that the lncRNA MLLT4-AS1 was downregulated in gastric cancer. Decreased expression of MLLT4-AS1 was associated with LN metastasis and a poor prognosis in patients with gastric cancer. These data suggest that MLLT4-AS1 is a potential biomarker for the diagnosis of gastric cancer.

\section{Acknowledgements}

This study was supported by the Medical Leading Project of Songjiang Commission of Health and Family Planning (grant no. 2011LX07), the Youth Scientific Research Fund of Shanghai Municipal Commission of Health and Family Planning (grant no. 20144Y0162), the Key Medical Specialties Fund of Shanghai (grant no. ZK2012A38), the Science and Technology Development Fund of Nanjing Medical University (grant no. 2016NJMU161) and the Shanghai Municipal Natural Science Foundation (grant no. 16ZR1432000).

\section{References}

1. Kamangar F, Dores GM and Anderson WF: Patterns of cancer incidence, mortality, and prevalence across five continents: Defining priorities to reduce cancer disparities in different geographic regions of the world. J Clin Oncol 24: 2137-2150, 2006.

2. Jemal A, Bray F, Center MM, Ferlay J, Ward E and Forman D: Global cancer statistics. CA Cancer J Clin 61: 69-90, 2011.

3. Hartgrink HH, Jansen EP, van Grieken NC and van de Velde CJ: Gastric cancer. Lancet 374: 477-490, 2009.

4. Mattick JS: Challenging the dogma: The hidden layer of non-protein-coding RNAs in complex organisms. Bioessays 25 930-939, 2003

5. Mattick JS: RNA regulation: A new genetics? Nat Rev Genet 5: 316-323, 2004.

6. Szymanski M, Barciszewska MZ, Erdmann VA and Barciszewski J: A new frontier for molecular medicine: Noncoding RNAs. Biochim Biophys Acta 1756: 65-75, 2005.

7. Prasanth KV and Spector DL: Eukaryotic regulatory RNAs: An answer to the 'genome complexity' conundrum. Genes Deve 21: 11-42, 2007.

8. Huarte M and Rinn JL: Large non-coding RNAs: Missing links in cancer? Hum Mol Genet 19: R152-R161, 2010.

9. Pauli A, Rinn JL and Schier AF: Non-coding RNAs as regulators of embryogenesis. Nat Rev Genet 12: 136-149, 2011.

10. Rinn JL, Kertesz M, Wang JK, Squazzo SL, Xu X, Brugmann SA, Goodnough LH, Helms JA, Farnham PJ, Segal E and Chang HY: Functional demarcation of active and silent chromatin domains in human HOX loci by noncoding RNAs. Cell 129: 1311-1323, 2007.
11. Khalil AM, Guttman M, Huarte M, Garber M, Raj A, Rivea Morales D, Thomas K, Presser A, Bernstein BE, van Oudenaarden A, et al: Many human large intergenic noncoding RNAs associate with chromatin-modifying complexes and affect gene expression. Proc Natl Acad Sci USA 106: 11667-11672, 2009.

12. Spitale RC, Tsai MC and Chang HY: RNA templating the epigenome: Long noncoding RNAs as molecular scaffolds. Epigenetics 6: 539-543, 2011.

13. Costa FF: Non-coding RNAs: Meet thy masters. Bioessays 32: 599-608, 2010.

14. Popov $\mathrm{N}$ and Gil J: Epigenetic regulation of the INK4b-ARF-INK4a locus: In sickness and in health. Epigenetics 5: 685-690, 2010

15. Calin GA, Liu CG, Ferracin M, Hyslop T, Spizzo R, Sevignani C, Fabbri M, Cimmino A, Lee EJ, Wojcik SE, et al: Ultraconserved regions encoding ncRNAs are altered in human leukemias and carcinomas. Cancer Cell 12: 215-229, 2007.

16. Sobin LH and Compton CC: TNM seventh edition: What's new, what's changed: Communication from the International Union Against Cancer and the American Joint Committee on Cancer. Cancer 116: 5336-5339, 2010.

17. Livak and Schmittgen: Analysis of relative gene expression data using real-time quantitative PCR and the $2-\Delta \Delta \mathrm{Ct}$ method. Methods 25: 402-408, 2001

18. Nault JC and Zucman-Rossi J: Genetics of hepatobiliary carcinogenesis. Semin Liver Dis 31: 173-187, 2011.

19. Kanwal R and Gupta S: Epigenetic modifications in cancer. Clin Genet 81: 303-311, 2012.

20. Oliveira C, Seruca R, Seixas M and Sobrinho-Simões M: The clinicopathological features of gastric carcinomas with microsatellite instability may be mediated by mutations of different 'target genes': A study of the TGFbeta RII, IGFII R and BAX genes. Am J Pathol 153: 1211-1219, 1998.

21. Dos Santos NR and Van Kessel AG: Chromosomal abnormalities: Detection and implications for cancer development. Anticancer Res 19: 4697-4714, 1999.

22. Ochi H, Douglass HO Jr and Sandberg AA: Cytogenetic studies in primary gastric cancer. Cancer Genet Cytogenet 22: 295-307, 1986.

23. Seruca R, Castedo S, Correia C, Gomes P, Carneiro F, Soares P, de Jong B and Sobrinho-Simões M: Cytogenetic findings in eleven gastric carcinomas. Cancer Genet Cytogenet 68: 42-48, 1993.

24. Panani AD, Ferti A, Malliaros S and Raptis S: Cytogenetic study of 11 gastric adenocarcinomas. Cancer Genet Cytogenet 81: 169-172, 1995.

25. Seruca R, Constancia M, Dossantos N, David L, Queimado L, Carvalho F and Carneiro F: Allele loss in human gastric carcinomas-relation to tumor progression and differentiation. Int J Oncol 7: 1159-1166, 1995.

26. Gleeson CM, Sloan JM, McGuigan JA, Ritchie AJ, Weber JL and Russell SE: Allelotype analysis of adenocarcinoma of the gastric cardia. Br J Cancer 76: 1455-1465, 1997.

27. Queimado L, Seruca R, Costa-Pereira A and Castedo S: Identification of two distinct regions of deletion at $6 \mathrm{q}$ in gastric carcinoma. Genes Chromosomes Cancer 14: 28-34, 1995.

28. Prensner JR and Chinnaiyan AM: The emergence of IncRNAs in cancer biology. Cancer Discov 1: 391-407, 2011.

29. Morris KV and Vogt PK: Long antisense non-coding RNAs and their role in transcription and oncogenesis. Cell Cycle 9: 2544-2547, 2010.

30. Xu Y, Chang R, Peng Z, Wang Y, Ji W, Guo J, Song L, Dai C, Wei $\mathrm{W}, \mathrm{Wu} \mathrm{Y}$, et al: Loss of polarity protein AF6 promotes pancreatic cancer metastasis by inducing Snail expression. Nat Commun 6: 7184, 2015.

31. Sun TT, Wang Y, Cheng H, Xiao HZ, Xiang JJ, Zhang JT, Yu SB, Martin TA, Ye L, Tsang LL, et al: Disrupted interaction between CFTR and AF-6/afadin aggravates malignant phenotypes of colon cancer. Biochim Biophys Acta 1843: 618-628, 2014

32. Yamamoto $\mathrm{T}$, Mori $\mathrm{T}$, Sawada M, Matsushima H, Ito $\mathrm{F}$, Akiyama M and Kitawaki J: Loss of AF-6/afadin induces cell invasion, suppresses the formation of glandular structures and might be a predictive marker of resistance to chemotherapy in endometrial cancer. BMC Cancer 15: 275, 2015.

33. Fournier G, Cabaud O, Josselin E, Chaix A, Adélaïde J,Isnardon D, Restouin A, Castellano R, Dubreuil P, Chaffanet M, et al: Loss of AF6/afadin, a marker of poor outcome in breast cancer, induces cell migration, invasiveness and tumor growth. Oncogene 30: 3862-3874, 2011. 